\title{
Assessment of fungicide spray schedules against turcicum leaf blight of maize caused by Exserohilum turcicum (Pass.) Leonard and Suggs
}

\author{
S. P. Meghana*, S. I. Harlapur, P.V. Patil and R. M. Kachapur \\ Department of Plant Pathology, College of Agriculture, Dharwad, University of Agricultural \\ Sciences, Dharwad-580005, Karnataka, India \\ *Corresponding author
}

A B S T R A C T

K e y w o r d s
Disease severity,
Exserohilum
turcicum, Fungicide
spray schedules,
Maize, Turcicum
leaf blight
Article Info
Accepted:
05 April 2020
Available Online:
10 May 2020

\section{Introduction}

Maize (Zea mays L.) is third most important cereal crop after rice and wheat. Maize can be grown under varied agro-climatic conditions as it has wider adaptability. It is globally recognized as "Miracle crop" and "Queen of cereals" because of its higher genetic yield potential compared to other cereals. The increase in interest of the consumers in
Turcicum leaf blight (TLB) caused by Exserohilum turcicum is a major foliar disease affecting maize. The aims of this study were to: assess tebuconazole (folicure $250 \mathrm{EC}$ ) spray schedules on disease severity of TLB, determine optimum growth stage of the crop for tebuconazole spray and amount of spray solution of fungicide to be applied for cost effective TLB management. The effect of fungicide spray schedules on TLB management was assessed in a field experiment during kharif 2018 at MARS, Dharwad. In this experiment, ten treatments were imposed with fungicide tebuconazole $250 \%$ EC @ $0.1 \%$ foliar spray at different growth stages of the crop irrespective of disease occurrence besides a treatment with unsprayed control. Data were recorded on disease severity. Eight time severity scores were used to calculate area under disease progress curve (AUDPC). Grain yield and yield components were measured after harvest. Finally economics of the treatments were carried out. The results of present investigation revealed that the disease severity was significantly reduced by different spray schedules. However, combined spray at the onset of disease and spray before tasseling (T8) was found effective in controlling TLB, which recorded significantly lowest disease severity (28.52 per cent) with 57.47 per cent reduction over control and AUDPC value of 915.75. 
India, Andhra Pradesh ranks first in production $(4.24 \mathrm{mt})$ followed by Karnataka (3.98 $\mathrm{mt})$ and Maharashtra (2.73 mt). In Karnataka it occupies an area of $1.17 \mathrm{~m}$ ha and production of $3.26 \mathrm{mt}$ and productivity of 2,700 kg ha-1 (Anon., 2017).

Globally turcicum leaf blight (TLB) disease has emerged as a constraint to maize production in many temperate and tropical environments. TLB is one of the ubiquitous foliar disease of maize. It is caused by the anamorph of the Deuteromycete, Exserohilum turcicum (Pass.) Leonard and Suggs. and the telomorph of the ascomycete, Setosphaeria turcica (Luttrell) Leonard and Suggs. First time, it was reported by Passerine (1876) in Perma, Italy, this was followed by a serious outbreak of TLB in Connecticut, New England in 1889 (Drechsler, 1923). The disease effect leads to the qualitative changes in the seed resulting in reduced germination capacity and decreased sugar content. The extent of yield losses depends on two factors i.e., stage of crop at which the infection occurs and the disease severity. If the disease appears before silking, the reduction of yield goes up to 40 per cent and the yield losses are minimal if infection is delayed until 6-8 weeks after silking. However the yield loss approaches up to 50 per cent when the disease is severe at 2-3 weeks after pollination, with the reduction in grain yield of 28 to 91 per cent (Nwanosike et al., 2015).

However for the management of TLB, the control measures like seed treatment, application of fungicides, use of resistant and tolerant genotypes have been recommended (Anon., 2004). Besides these control measures, TLB has continued to be a major threat in maize production. In this view of increasing severity of TLB the present experiment was carried out to get the information on the stage of crop at which fungicides need to be applied and quantity of spray solution to be used is lacking. So in order to assess the economic management and to get high benefit cost ratio, this study was undertaken on the assessment of fungicide spray schedule for cost effective TLB management.

\section{Materials and Methods}

The field experiment was conducted at Main Agricultural Research Station, University of Agricultural Sciences, Dharwad during kharif 2018. The experiment was laid out in a randomized complete block design having three replications and eleven treatments with the spacing $60 \mathrm{~cm} \times 20 \mathrm{~cm}$. The gross plot size was 14.4 sq. $\mathrm{m}$ and net plot size was 9.6 sq. m. The susceptible cultivar P 3501 was sown. The Exserohilum turcicum culture mass multiplied in sorghum grains was artificially inoculated on all the treatments when crop was 30 to 40 days old following whorl- drop method of inoculation. Ten treatments were imposed with fungicide tebuconazole $250 \%$ EC @ $0.1 \%$ foliar spray besides a treatment with unsprayed control at different growth stages of the crop. The details of the treatments are given in Table 1.

\section{Data recording}

The severity of turcicum leaf blight was recorded by scoring randomly selected individual ten plants in each treatment at weekly interval starting from the onset of disease till physiological maturity was recorded as per the 0-9 rating scale (Anon., 2016). Further the PDI was calculated using the following formula given by Wheeler (1969).

Sum of all the individual

PDI $=\frac{\text { disease ratings }}{\text { Total number of plants observed Maximum }}$
grade


Further, PDI values were used to calculate the area under disease progress curve (AUDPC) using the following formula given by Wilcoxson et al., (1975).

$\operatorname{AUDPC}=\sum_{\mathrm{i}=1}^{\mathrm{k}}\left(\frac{1}{2}\left(\mathrm{~S}_{\mathrm{i}}+\mathrm{Si}_{-1}\right) \mathrm{d}\right)$

Where, $\mathrm{S}_{\mathrm{i}}=$ Disease severity at the end of time

$\mathrm{S}_{\mathrm{i}-1}=$ Number of successive evaluations of blight

$\mathrm{d}=$ Interval between two evaluations

Grain yield, fodder yield, shelling per cent, hundred seed weight and economics of the treatments were recorded in individual plots after the harvest and these data was further converted into hectare.

\section{Results and Discussion}

The results of present investigation revealed that the disease severity was significantly reduced by different spray schedules. However, combined spray at the onset of disease and spray before tasseling $\left(\mathrm{T}_{8}\right)$ was found effective in controlling TLB, which recorded significantly lowest disease severity (28.52 per cent) with 57.47 per cent reduction over control and AUDPC value of 915.75. This treatment was statistically on par with $\mathrm{T}_{9}$ i.e., combined spray at the onset of disease and spray after tasseling with disease severity of 30.37 per cent and AUDPC of 971.91. While prophylactic spray $\left(\mathrm{T}_{1}\right)$ showed the maximum disease severity (56.72 per cent) with 15.42 per cent reduction over control and AUDPC value of 1316.66. Whereas spray before tasseling $\left(\mathrm{T}_{3}\right)$ and combined prophylactic spray and spray at the onset of disease $\left(T_{5}\right)$ were statistically on par with each other with disease severity of 52.26 per cent and 51.52 per cent, respectively). The AUDPC values of $T_{3}$ and $T_{5}$ were recorded as
1263.21 and 1241.76, respectively. Among remaining treatments the best treatment was $\mathrm{T}_{10}$ i.e., combined spray before tasseling and spray after tasseling with the disease severity of 35.27 per cent and AUDPC of 1030.34 followed by $\mathrm{T}_{6}$ i.e., combined prophylactic spray and spray before tasseling with disease severity of 37.53 per cent and AUDPC value of 1066.94. However, spray after tasseling $\left(\mathrm{T}_{4}\right)$ and combined prophylactic spray and spray after tasseling $\left(\mathrm{T}_{7}\right)$ were recorded with disease severity of 44.50 per cent and 48.87 per cent and AUDPC values of 1159.25 and 1222.75 respectively as indicated in Table $1 \mathrm{a}$, 1b, Fig. 1, Fig. 2, Fig. 3 and Plate 1.

With respect to yield parameters, the combined spray at the onset of disease and spray before tasseling $\left(\mathrm{T}_{8}\right)$ has recorded significantly higher grain yield (66.31 q/ha) with 26.99 per cent increase over control and fodder yield (13.53 t/ha) with 38.65 per cent increase over control. This treatment was followed by combined spray at the onset of disease and spray after tasseling $\left(\mathrm{T}_{9}\right)$ with grain yield $(63.48 \mathrm{q} / \mathrm{ha})$ and fodder yield $(12.53 \mathrm{t} / \mathrm{ha})$. While prophylactic spray $\left(\mathrm{T}_{1}\right)$ was recorded minimum grain yield (51.61 q/ha) with 6.18 per cent per cent increase over control and fodder yield (9.42 t/ha) with 11.89 per cent increase over control. Whereas spray before tasseling $\left(\mathrm{T}_{3}\right)$ and combined prophylactic spray and spray at the onset disease $\left(T_{5}\right)$ were statistically on par with each other with grain yield of $53.95 \mathrm{q} / \mathrm{ha}$ and $54.00 \mathrm{q} / \mathrm{ha}$, respectively and fodder yield of (10.30 t/ha and 10.47 t/ha respectively. Among remaining treatments, combined spray before tasseling and spray after tasseling $\left(\mathrm{T}_{10}\right)$ was found to be best with the recorded grain yield of $61.25 \mathrm{q} / \mathrm{ha}$ and fodder yield of 11.91 $\mathrm{t} /$ ha followed by $\mathrm{T}_{6}$ i.e., combined prophylactic spray and spray before tasseling with grain yield of $59.23 \mathrm{q} / \mathrm{ha}$ and fodder yield of $11.54 \mathrm{t} / \mathrm{ha}$. 
Table.1a Assessment of fungicidal spray schedules for the management of turcicum leaf blight

\begin{tabular}{|c|c|c|c|c|c|c|c|c|c|c|}
\hline $\begin{array}{l}\text { Treatment } \\
\text { No. }\end{array}$ & Treatment details & $\begin{array}{l}\text { Per cent } \\
\text { disease } \\
\text { index }\end{array}$ & $\begin{array}{l}\text { Per cent } \\
\text { reduction } \\
\text { over } \\
\text { control }\end{array}$ & $\begin{array}{l}\text { Grain } \\
\text { yield } \\
\text { (q/ha) }\end{array}$ & $\begin{array}{c}\text { Per cent } \\
\text { increase } \\
\text { over } \\
\text { control }\end{array}$ & $\begin{array}{l}\text { Fodder } \\
\text { yield } \\
\text { (t/ha) }\end{array}$ & $\begin{array}{c}\text { Per cent } \\
\text { increase } \\
\text { over } \\
\text { control }\end{array}$ & $\begin{array}{l}\text { Shelling } \\
\text { per cent }\end{array}$ & $\begin{array}{l}100 \text { seed } \\
\text { weight } \\
\text { (g) }\end{array}$ & $\begin{array}{c}\text { Spray } \\
\text { solution } \\
\text { required } \\
\text { (l/ha) }\end{array}$ \\
\hline $\mathrm{T}_{1}$ & Prophylactic spray (30 DAS) & $\begin{array}{c}56.72 \\
(48.89)^{*}\end{array}$ & 15.42 & 51.61 & 06.18 & 09.42 & 11.89 & 83.86 & 29.69 & 235 \\
\hline $\mathrm{T}_{2}$ & $\begin{array}{l}\text { Spray at the onset of disease } \\
\text { (40 DAS) }\end{array}$ & $\begin{array}{c}46.34 \\
(42.89)\end{array}$ & 30.90 & 56.33 & 14.04 & 11.11 & 25.29 & 84.89 & 31.77 & 325 \\
\hline $\mathrm{T}_{3}$ & $\begin{array}{l}\text { Spray before tasseling ( } 60 \\
\text { DAS) }\end{array}$ & $\begin{array}{c}52.26 \\
(46.31)\end{array}$ & 22.07 & 53.95 & 10.25 & 10.30 & 19.42 & 83.79 & 30.21 & 425 \\
\hline $\mathrm{T}_{4}$ & Spray after tasseling (70 DAS) & $\begin{array}{c}44.50 \\
(41.83)\end{array}$ & 33.64 & 57.06 & 15.14 & 11.39 & 27.13 & 84.98 & 32.29 & 510 \\
\hline $\mathrm{T}_{5}$ & $\mathrm{~T}_{1}+\mathrm{T}_{2}$ & $\begin{array}{c}51.52 \\
(45.87)\end{array}$ & 23.17 & 54.00 & 10.33 & 10.47 & 20.73 & 83.94 & 30.73 & 560 \\
\hline $\mathrm{T}_{6}$ & $\mathrm{~T}_{1}+\mathrm{T}_{3}$ & $\begin{array}{c}37.53 \\
(37.61)\end{array}$ & 44.03 & 59.23 & 18.13 & 11.54 & 11.52 & 85.10 & 32.81 & 660 \\
\hline $\mathrm{T}_{7}$ & $\mathrm{~T}_{1}+\mathrm{T}_{4}$ & $\begin{array}{c}48.87 \\
(44.35)\end{array}$ & 27.12 & 54.62 & 11.35 & 10.58 & 21.55 & 84.63 & 31.25 & 745 \\
\hline $\mathrm{T}_{8}$ & $\mathrm{~T}_{2}+\mathrm{T}_{3}$ & $\begin{array}{c}28.52 \\
(32.23)\end{array}$ & 57.47 & 66.31 & 26.99 & 13.53 & 38.65 & 85.67 & 35.42 & 750 \\
\hline $\mathrm{T}_{9}$ & $\mathrm{~T}_{2}+\mathrm{T}_{4}$ & $\begin{array}{c}30.37 \\
(33.33)\end{array}$ & 54.71 & 63.48 & 23.72 & 12.53 & 33.76 & 85.46 & 34.38 & 835 \\
\hline $\mathrm{T}_{10}$ & $\mathrm{~T}_{3}+\mathrm{T}_{4}$ & $\begin{array}{c}35.27 \\
(36.22)\end{array}$ & 47.41 & 61.25 & 20.95 & 11.91 & 30.31 & 85.38 & 33.33 & 935 \\
\hline $\mathrm{T}_{11}$ & Unsprayed control & $\begin{array}{c}67.06 \\
(55.00)\end{array}$ & - & 48.42 & - & 8.30 & - & 82.85 & 29.17 & - \\
\hline & $\begin{array}{c}\text { S.Em. } \pm \\
\text { C.D. @ } 5 \% \\
\text { C.V. }(\%)\end{array}$ & $\begin{array}{l}2.01 \\
5.92 \\
8.23\end{array}$ & & $\begin{array}{c}1.66 \\
4.91 \\
15.26\end{array}$ & & $\begin{array}{c}0.58 \\
1.70 \\
19.07\end{array}$ & & $\begin{array}{l}1.36 \\
4.01 \\
6.91\end{array}$ & $\begin{array}{l}0.82 \\
2.43 \\
4.66\end{array}$ & \\
\hline
\end{tabular}

* Arcsine transformed values DAS - Days after sowing 
Table.1b Per cent disease index and computed area under disease progress curve (AUDPC) in different spray schedules

\begin{tabular}{|c|c|c|c|c|c|c|c|c|c|c|}
\hline \multirow{2}{*}{$\begin{array}{l}\text { Treatmen } \\
\text { t No. }\end{array}$} & \multirow[t]{2}{*}{ Treatment details } & \multicolumn{8}{|c|}{ Per cent disease index at weekly intervals } & \multirow[b]{2}{*}{$\begin{array}{c}\text { AUDPC } \\
\text { value }\end{array}$} \\
\hline & & 42 DAS & $\begin{array}{c}49 \\
\text { DAS }\end{array}$ & $\begin{array}{c}56 \\
\text { DAS }\end{array}$ & $\begin{array}{c}63 \\
\text { DAS }\end{array}$ & $\begin{array}{c}70 \\
\text { DAS }\end{array}$ & $\begin{array}{c}77 \\
\text { DAS }\end{array}$ & $\begin{array}{c}84 \\
\text { DAS }\end{array}$ & 91 DAS & \\
\hline $\mathbf{T}_{1}$ & Prophylactic spray (30 DAS) & $\begin{array}{c}5.89 \\
(13.81)^{*}\end{array}$ & $\begin{array}{c}11.02 \\
(19.38)\end{array}$ & $\begin{array}{c}15.84 \\
(23.39)\end{array}$ & $\begin{array}{c}20.86 \\
(27.16)\end{array}$ & $\begin{array}{c}26.61 \\
(31.04)\end{array}$ & $\begin{array}{c}33.35 \\
(35.27)\end{array}$ & $\begin{array}{c}49.94 \\
(44.97)\end{array}$ & $\begin{array}{c}56.72 \\
(48.89)\end{array}$ & 1316.66 \\
\hline $\mathbf{T}_{2}$ & Spray at the onset of disease (40 DAS) & $\begin{array}{c}5.56 \\
(13.46)\end{array}$ & $\begin{array}{c}9.07 \\
(17.49)\end{array}$ & $\begin{array}{c}13.16 \\
(21.25)\end{array}$ & $\begin{array}{c}16.96 \\
(24.28)\end{array}$ & $\begin{array}{c}22.35 \\
(28.20)\end{array}$ & $\begin{array}{c}24.40 \\
(29.60)\end{array}$ & $\begin{array}{c}39.54 \\
(38.96)\end{array}$ & $\begin{array}{c}46.34 \\
(42.89)\end{array}$ & 1183.21 \\
\hline $\mathbf{T}_{\mathbf{3}}$ & Spray before tasselling (60 DAS) & $\begin{array}{c}5.83 \\
(13.84)\end{array}$ & $\begin{array}{c}10.80 \\
(19.18)\end{array}$ & $\begin{array}{c}14.86 \\
(22.65)\end{array}$ & $\begin{array}{c}19.67 \\
(26.29)\end{array}$ & $\begin{array}{c}25.75 \\
(30.48)\end{array}$ & $\begin{array}{c}31.35 \\
(34.50)\end{array}$ & $\begin{array}{c}48.60 \\
(44.20)\end{array}$ & $\begin{array}{c}52.26 \\
(46.31)\end{array}$ & 1263.21 \\
\hline $\mathbf{T}_{4}$ & Spray after tasselling (70 DAS) & $\begin{array}{c}5.39 \\
(13.38)\end{array}$ & $\begin{array}{c}8.68 \\
(17.04)\end{array}$ & $\begin{array}{c}12.51 \\
(20.65)\end{array}$ & $\begin{array}{c}15.90 \\
(23.46)\end{array}$ & $\begin{array}{c}21.95 \\
(27.93)\end{array}$ & $\begin{array}{c}23.70 \\
(29.13)\end{array}$ & $\begin{array}{c}38.65 \\
(38.44)\end{array}$ & $\begin{array}{c}44.50 \\
(41.83)\end{array}$ & 1159.25 \\
\hline $\mathbf{T}_{\mathbf{5}}$ & $\mathrm{T}_{1}+\mathrm{T}_{2}$ & $\begin{array}{c}5.78 \\
(13.26)\end{array}$ & $\begin{array}{c}10.59 \\
(18.85)\end{array}$ & $\begin{array}{c}14.41 \\
(22.30)\end{array}$ & $\begin{array}{c}18.83 \\
(25.70)\end{array}$ & $\begin{array}{c}24.81 \\
(29.84)\end{array}$ & $\begin{array}{c}30.95 \\
(33.80)\end{array}$ & $\begin{array}{c}47.67 \\
(43.66)\end{array}$ & $\begin{array}{c}51.52 \\
(45.87)\end{array}$ & 1241.76 \\
\hline $\mathbf{T}_{6}$ & $\mathrm{~T}_{1}+\mathrm{T}_{3}$ & $\begin{array}{c}5.23 \\
(13.20)\end{array}$ & $\begin{array}{c}8.23 \\
(16.62)\end{array}$ & $\begin{array}{c}11.05 \\
(19.41)\end{array}$ & $\begin{array}{c}13.81 \\
(21.79)\end{array}$ & $\begin{array}{c}20.70 \\
(27.05)\end{array}$ & $\begin{array}{c}21.87 \\
(27.88)\end{array}$ & $\begin{array}{c}28.97 \\
(32.56)\end{array}$ & $\begin{array}{c}37.53 \\
(37.61)\end{array}$ & 1066.94 \\
\hline $\mathbf{T}_{7}$ & $\mathrm{~T}_{1}+\mathrm{T}_{4}$ & $\begin{array}{c}5.75 \\
(13.87)\end{array}$ & $\begin{array}{c}9.83 \\
(18.24)\end{array}$ & $\begin{array}{c}13.33 \\
(21.38)\end{array}$ & $\begin{array}{c}17.94 \\
(25.04)\end{array}$ & $\begin{array}{c}23.77 \\
(29.17)\end{array}$ & $\begin{array}{c}30.06 \\
(33.25)\end{array}$ & $\begin{array}{c}45.23 \\
(42.26)\end{array}$ & $\begin{array}{c}48.87 \\
(44.35)\end{array}$ & 1222.75 \\
\hline $\mathbf{T}_{8}$ & $\mathrm{~T}_{2}+\mathrm{T}_{3}$ & $\begin{array}{c}5.05 \\
(12.37)\end{array}$ & $\begin{array}{c}7.49 \\
(15.83)\end{array}$ & $\begin{array}{c}8.37 \\
(16.81)\end{array}$ & $\begin{array}{c}10.08 \\
(18.50)\end{array}$ & $\begin{array}{c}15.93 \\
(23.51)\end{array}$ & $\begin{array}{c}17.79 \\
(24.95)\end{array}$ & $\begin{array}{c}21.70 \\
(27.77)\end{array}$ & $\begin{array}{c}28.52 \\
(31.23)\end{array}$ & 915.75 \\
\hline $\mathbf{T}_{9}$ & $\mathrm{~T}_{2}+\mathrm{T}_{4}$ & $\begin{array}{c}5.12 \\
(12.96)\end{array}$ & $\begin{array}{c}7.68 \\
(16.03)\end{array}$ & $\begin{array}{c}9.00 \\
(17.46)\end{array}$ & $\begin{array}{c}11.06 \\
(19.42)\end{array}$ & $\begin{array}{c}16.60 \\
(23.93)\end{array}$ & $\begin{array}{c}18.96 \\
(25.81)\end{array}$ & $\begin{array}{c}24.92 \\
(29.95)\end{array}$ & $\begin{array}{c}30.37 \\
(33.33)\end{array}$ & 971.91 \\
\hline $\mathbf{T}_{10}$ & $\mathrm{~T}_{3}+\mathrm{T}_{4}$ & $\begin{array}{c}5.19 \\
(12.85)\end{array}$ & $\begin{array}{c}8.03 \\
(16.42)\end{array}$ & $\begin{array}{c}9.67 \\
(18.08)\end{array}$ & $\begin{array}{c}11.33 \\
(19.63)\end{array}$ & $\begin{array}{c}18.78 \\
(25.62)\end{array}$ & $\begin{array}{c}20.57 \\
(26.97)\end{array}$ & $\begin{array}{c}28.76 \\
(32.43)\end{array}$ & $\begin{array}{c}35.27 \\
(36.22)\end{array}$ & 1030.34 \\
\hline $\mathbf{T}_{11}$ & Unsprayed control & $\begin{array}{c}5.94 \\
(13.84)\end{array}$ & $\begin{array}{c}12.79 \\
(20.83)\end{array}$ & $\begin{array}{c}17.85 \\
(24.96)\end{array}$ & $\begin{array}{c}22.91 \\
(28.59)\end{array}$ & $\begin{array}{c}30.99 \\
(33.82)\end{array}$ & $\begin{array}{c}41.94 \\
(40.36)\end{array}$ & $\begin{array}{c}55.17 \\
(47.97)\end{array}$ & $\begin{array}{c}67.06 \\
(55.01)\end{array}$ & 1445.68 \\
\hline & S.Em. \pm & 0.63 & 0.96 & 0.59 & 0.48 & 0.77 & 0.90 & 1.42 & 2.01 & \\
\hline & C.D.@ $5 \%$ & 1.86 & 2.01 & 1.73 & 1.42 & 2.28 & 2.65 & 4.20 & 5.92 & \\
\hline
\end{tabular}


Table.1c Economics of different fungicidal spray schedules for the management of turicum leaf blight of maize

\begin{tabular}{|c|c|c|c|c|c|c|c|}
\hline $\begin{array}{l}\text { Treatment } \\
\text { No. }\end{array}$ & Treatment details & $\begin{array}{c}\text { Per cent } \\
\text { disease } \\
\text { index }\end{array}$ & $\begin{array}{l}\text { Grain yield } \\
\text { (q/ha) }\end{array}$ & $\begin{array}{c}\text { Cost of } \\
\text { cultivation } \\
\text { (Rs/ha) }\end{array}$ & $\begin{array}{l}\text { Gross } \\
\text { returns } \\
\text { (Rs/ha) }\end{array}$ & $\begin{array}{l}\text { Net returns } \\
\quad(\mathrm{Rs} / \mathrm{ha})\end{array}$ & $\mathrm{B}: \mathrm{C}$ ratio \\
\hline $\mathrm{T}_{1}$ & Prophylactic spray (30 DAS) & $\begin{array}{c}56.72 \\
(48.89)^{*}\end{array}$ & 51.61 & 53,541 & 93,414 & 39,873 & 1.74 \\
\hline $\mathrm{T}_{2}$ & Spray at the onset of disease (40DAS) & $\begin{array}{c}46.34 \\
(42.89)\end{array}$ & 56.33 & 53,646 & $1,10,957$ & 57,311 & 2.07 \\
\hline $\mathrm{T}_{3}$ & Spray before tasseling (60DAS) & $\begin{array}{c}52.26 \\
(46.31)\end{array}$ & 53.95 & 53,673 & 97,650 & 43,977 & 1.82 \\
\hline $\mathrm{T}_{4}$ & Spray after tasseling (70 DAS) & $\begin{array}{c}44.50 \\
(41.83)\end{array}$ & 57.06 & 53,715 & $1,03,279$ & 49,564 & 1.92 \\
\hline $\mathrm{T}_{5}$ & $\mathrm{~T}_{1}+\mathrm{T}_{2}$ & $\begin{array}{c}51.52 \\
(45.87)\end{array}$ & 54.00 & 54,104 & 97,740 & 43,636 & 1.81 \\
\hline $\mathrm{T}_{6}$ & $\mathrm{~T}_{1}+\mathrm{T}_{3}$ & $\begin{array}{c}37.53 \\
(37.61)\end{array}$ & 59.23 & 54,139 & 97,740 & 53,067 & 1.98 \\
\hline $\mathrm{T}_{7}$ & $\mathrm{~T}_{1}+\mathrm{T}_{4}$ & $\begin{array}{c}48.87 \\
(44.35)\end{array}$ & 54.62 & 54,154 & 98,862 & 44,708 & 1.82 \\
\hline $\mathrm{T}_{8}$ & $\mathrm{~T}_{2}+\mathrm{T}_{3}$ & $\begin{array}{c}28.52 \\
(32.23)\end{array}$ & 66.31 & 54,668 & $1,20,021$ & 65,353 & 2.19 \\
\hline $\mathrm{T}_{9}$ & $\mathrm{~T}_{2}+\mathrm{T}_{4}$ & $\begin{array}{c}30.37 \\
(33.33)\end{array}$ & 63.48 & 54,668 & $1,14,899$ & 60,231 & 2.10 \\
\hline $\mathrm{T}_{10}$ & $\mathrm{~T}_{3}+\mathrm{T}_{4}$ & $\begin{array}{c}35.27 \\
(36.22)\end{array}$ & 61.25 & 55,127 & $1,10,863$ & 55,736 & 2.01 \\
\hline $\mathrm{T}_{11}$ & Unsprayed control & $\begin{array}{c}67.06 \\
(55.00)\end{array}$ & 48.42 & 52,553 & 87,640 & 35,087 & 1.67 \\
\hline & $\begin{array}{c}\text { S.Em. } \pm \\
\text { C.D. @ } 5 \% \\
\text { C.V. }(\%)\end{array}$ & $\begin{array}{l}2.01 \\
5.92 \\
8.23\end{array}$ & $\begin{array}{c}1.66 \\
4.91 \\
15.26\end{array}$ & & & & \\
\hline
\end{tabular}


Plate.1 Severity of turcicum leaf blight under different spray schedules

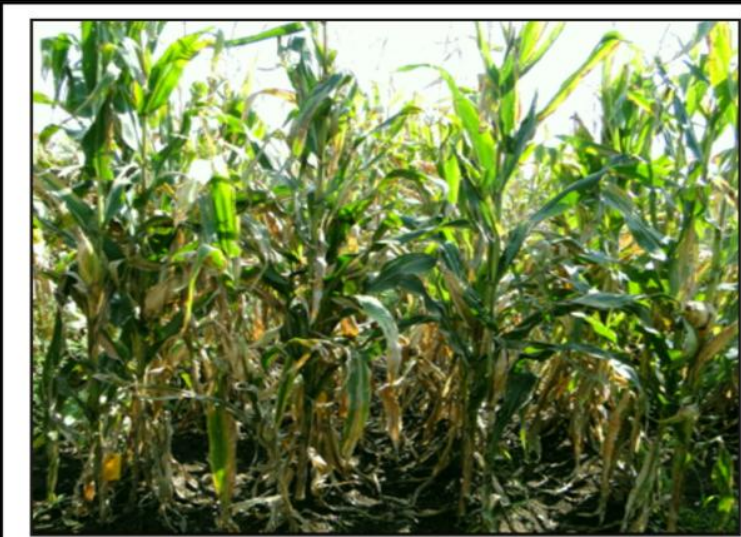

a) $T_{8}$ - Spray at the onset of the disease $\left(T_{2}\right)$ + Spray before tasseling $\left(T_{3}\right)$

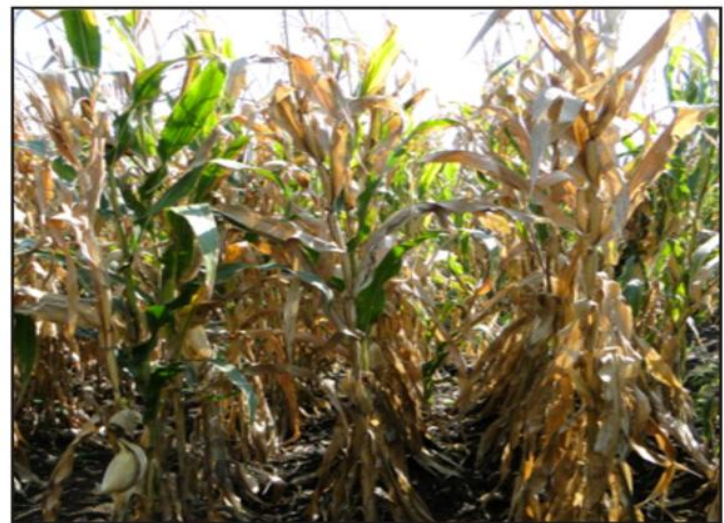

c) $T_{1}$ - Prophylactic spray

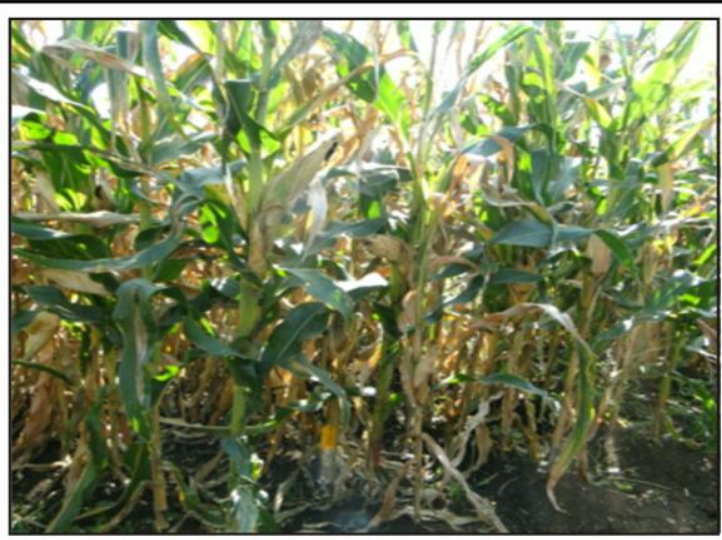

b) $T_{9}$ - Spray at the onset of the disease $\left(T_{2}\right)$ + Spray after tasseling $\left(T_{4}\right)$

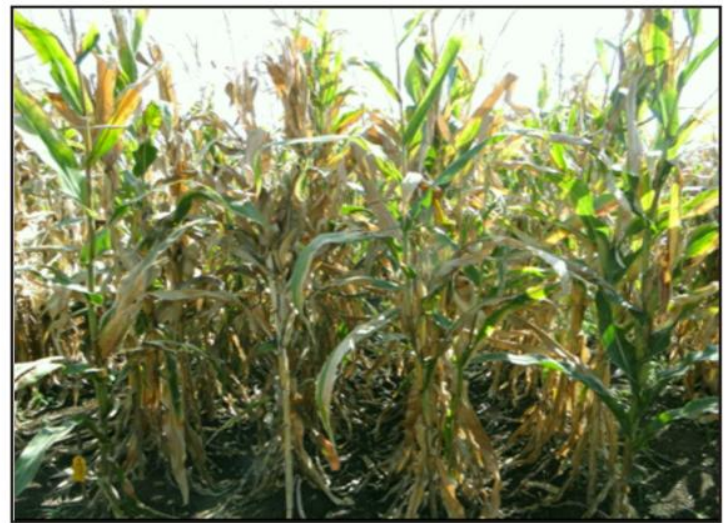

d) $T_{3}$ - Spray before tasseling

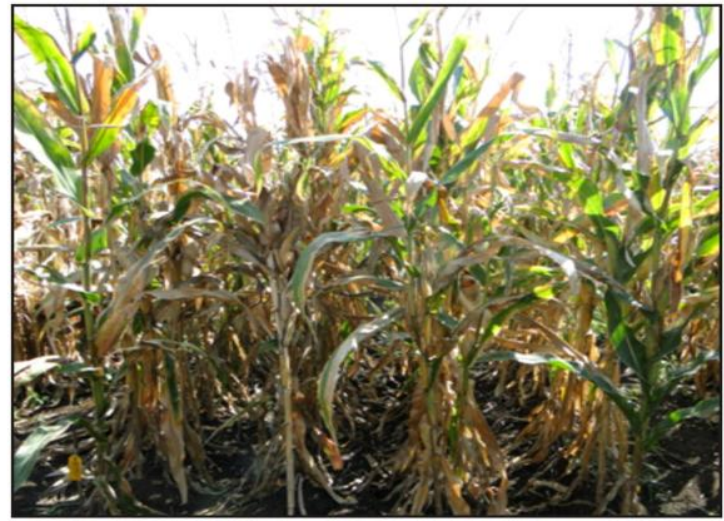

e) $T_{11}$ - Untreated control 


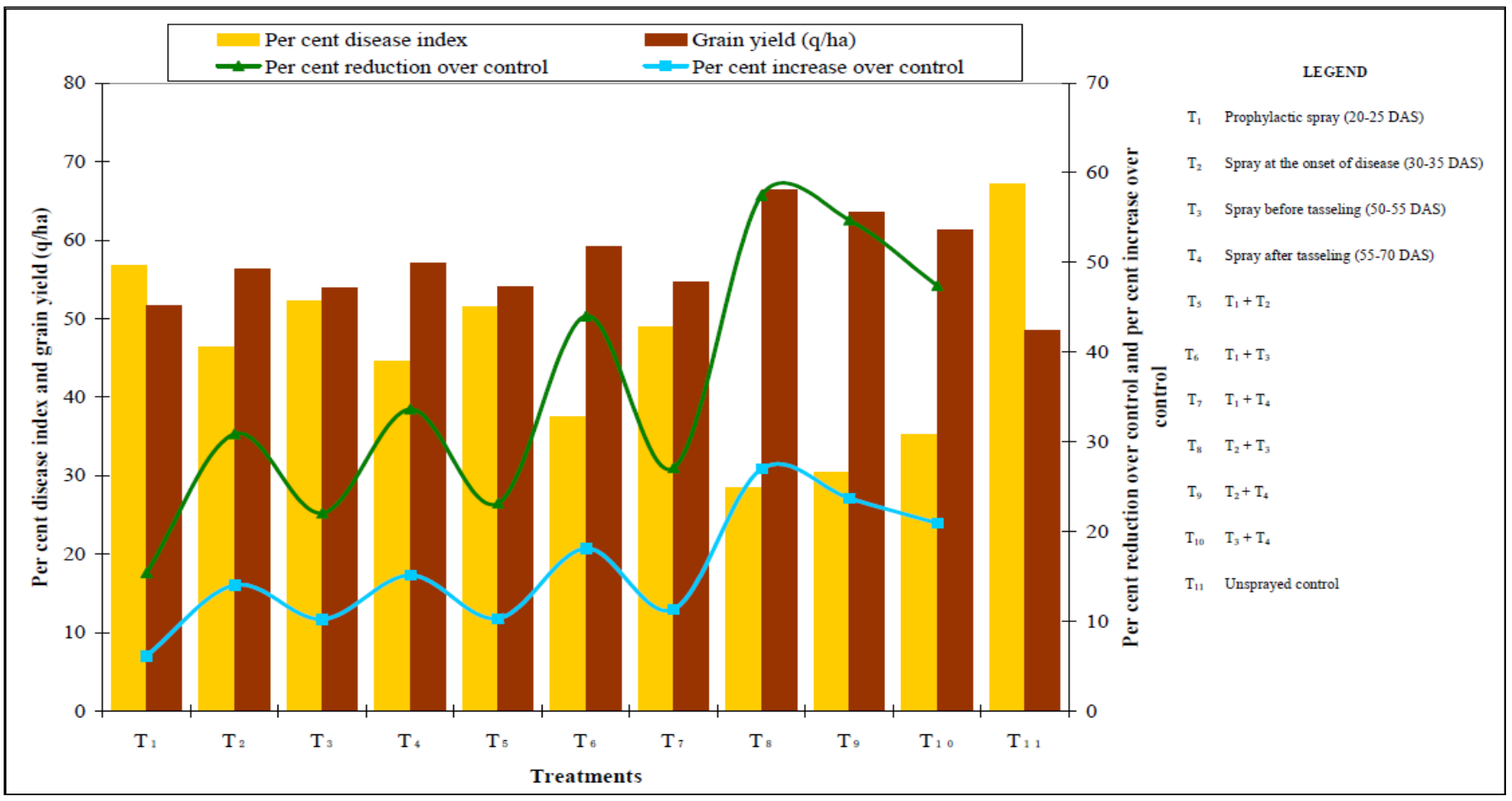

Fig.1 Influence of different spray schedules on PDI and grain yield

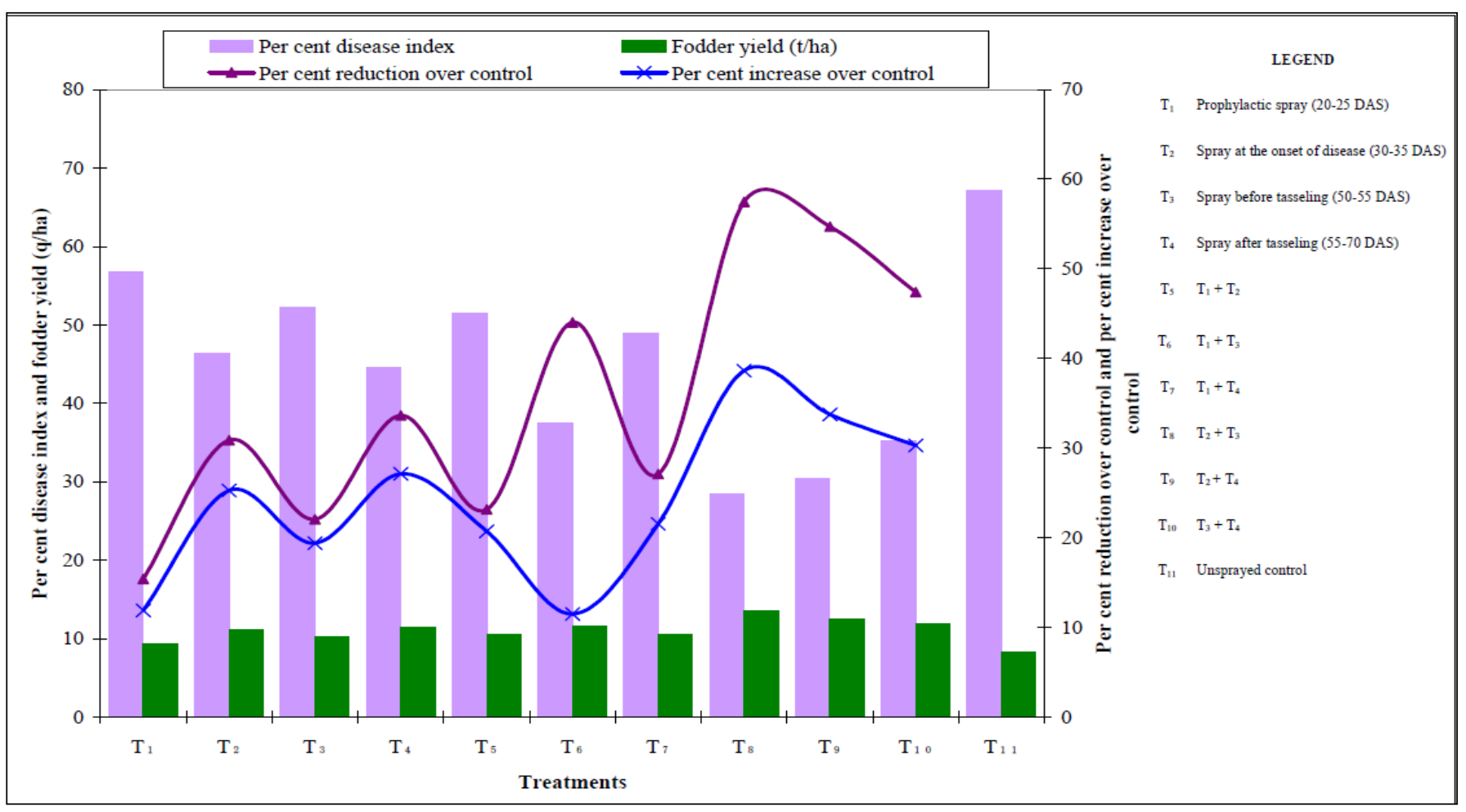

Fig.2 Influence of different spray schedules on PDI and fodder yield 


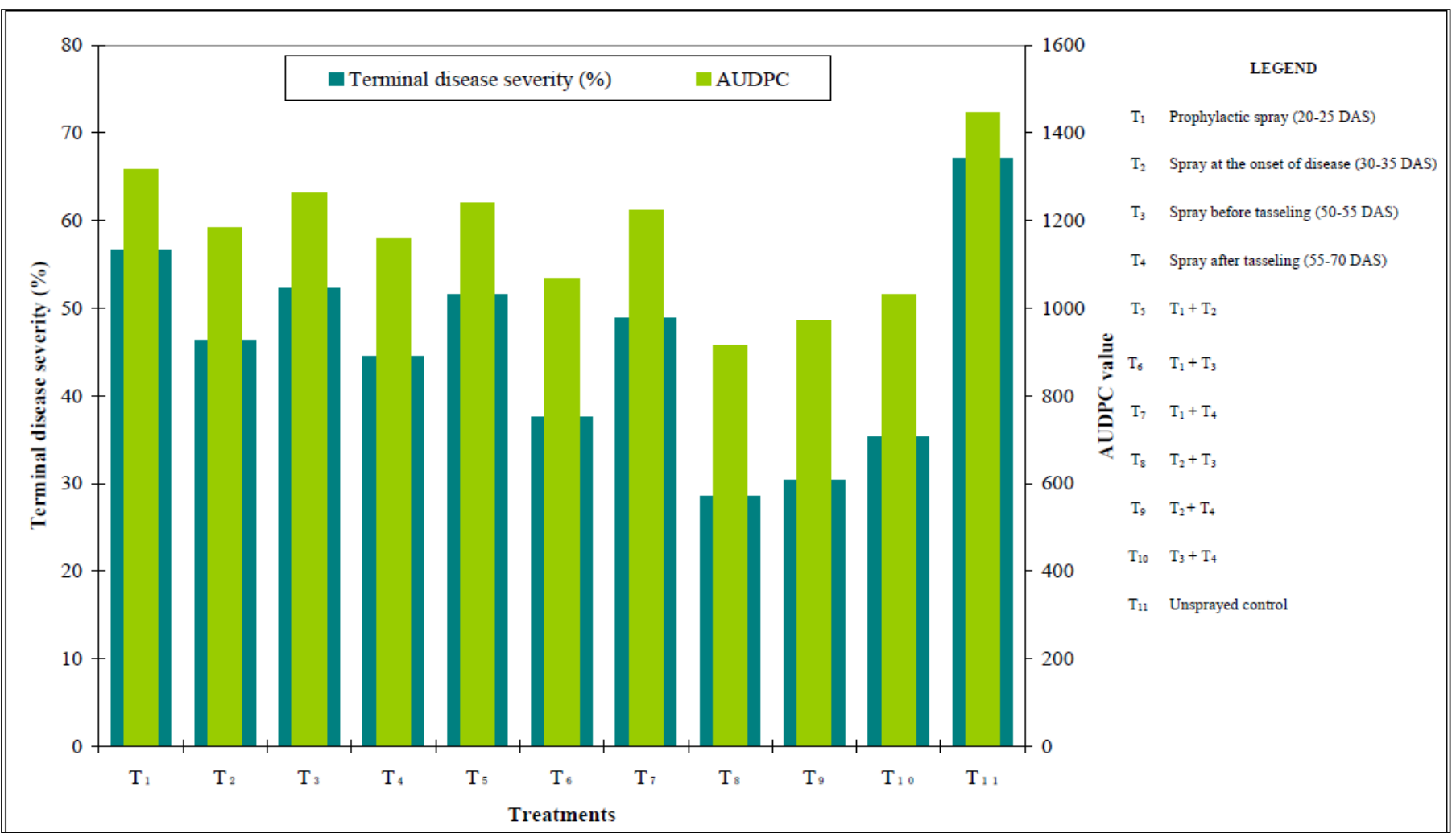

Fig.3 Influence of different spray schedules on terminal disease severity and AUDPC

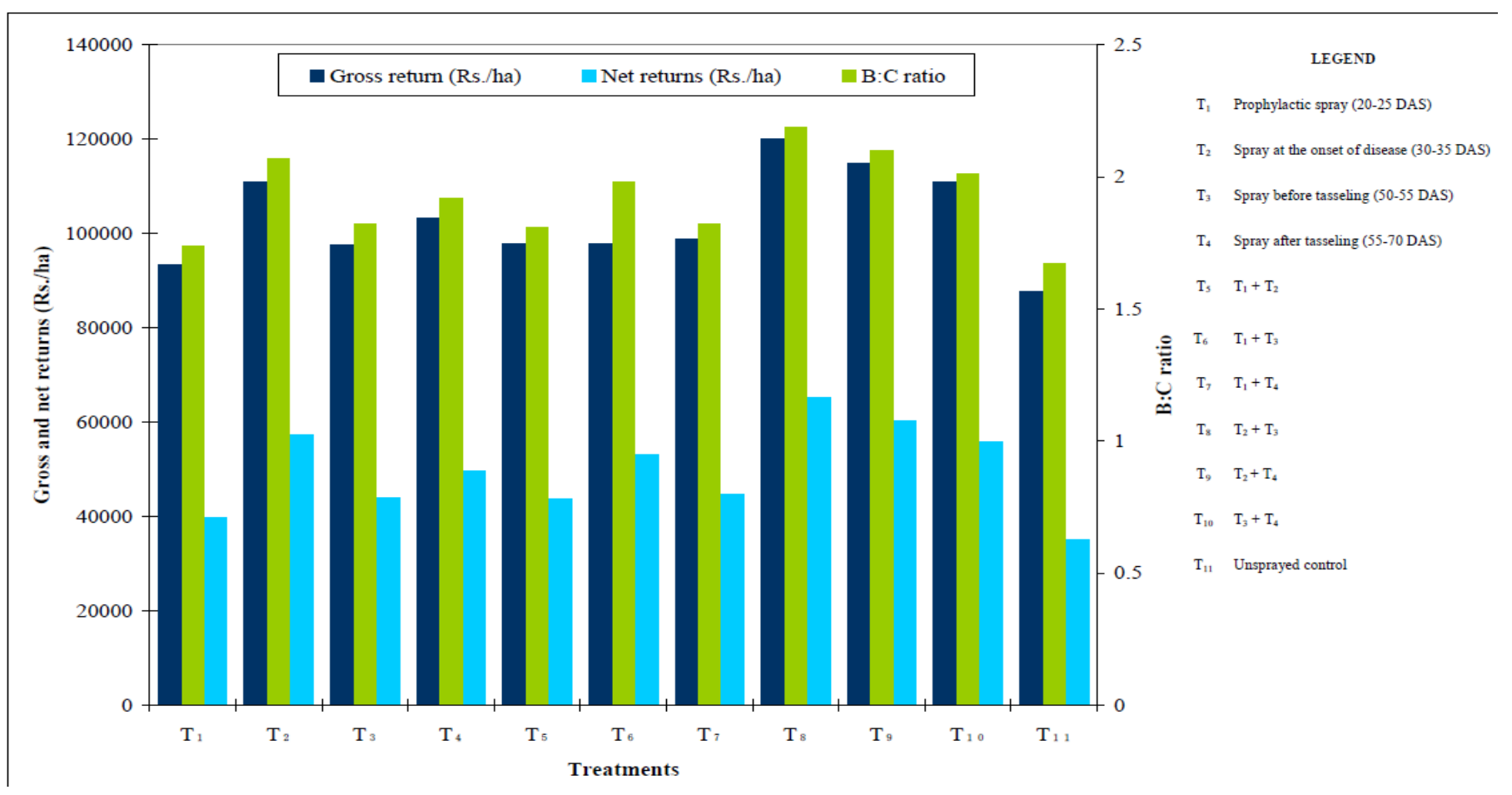

Fig.4 Economics of different spray schedules for the management of turcicum leaf blight 
However, spray after tasseling $\left(\mathrm{T}_{4}\right)$ and combined prophylactic spray and spray after tasseling $\left(\mathrm{T}_{7}\right)$ were recorded with grain yield of $57.06 \mathrm{q} / \mathrm{ha}$ and $54.62 \mathrm{q} / \mathrm{ha}$, respectively and fodder yield of $11.39 \mathrm{t} / \mathrm{ha}$ and $10.58 \mathrm{t} / \mathrm{ha}$ respectively, as indicated in Table 1a .

With respect to economics, combined spray at the onset of disease and spray before tasseling $\left(\mathrm{T}_{8}\right)$ recorded the maximum benefit cost ratio (2.19) followed by $\mathrm{T}_{9}$ i.e., combined spray at the onset of disease and spray after tasseling with B:C ratio of (2.10) compared to untreated control (1.67) as indicated in Table 1c and Fig. 4.

In conclusion, among different spray schedules, the spray at the onset on the disease and spray before tasseling was found effective in reducing the disease severity (28.52 per cent) and AUDPC (915.75) with maximum grain yield $(66.31 \mathrm{q} / \mathrm{ha})$, fodder yield (13.53 t/ha) and benefit cost ratio (2.19). These results are helpful for the efficient use of chemicals at the recommended dose for the sustainable management and helps in avoiding the excess use of chemicals and fungicidal toxicity.

\section{Acknowledgement}

The author wishes to thank Professor S. I. Harlapur, University of Agricultural Sciences, Dharwad, for his sustained interest in this work and the preparation of this paper.

\section{References}

Anonymous. 2004. Annual progress report
(2004 - 2005). All India Coordinated Maize Improvement Project, Indian Council of Agricultural Research, Indian Institute of Maize Research, Ludhiana. pp. 80-83.

Anonymous. 2016. Annual progress report (2016 - 2017). All India Coordinated Maize Improvement Project, Indian Council of Agricultural Research, Indian Institute of Maize Research, Ludhiana. pp. 214-216.

Anonymous. 2017. Annual progress report (2017 - 2018). All India Coordinated Maize Improvement Project, Indian Council of Agricultural Research, Indian Institute of Maize Research, Ludhiana. pp. 1-5.

Drechsler, C. 1923. Some graminicolous species of Helminthosporium. J. Agri. Res., 24: 641-739.

Nwanosike, M. R. O., Mabagala, R. B. and Kusolwa, P. M. 2015. Disease intensity and distribution of Exserohilum turcicum incitant of northern leaf blight of maize in Tanzania. Int. J. Pure App. Biosci., 3 (5): 1-13.

Passerini, 1876. Lanebbia Delgranotur Co. Bol. Comiz, Agriculture. Parmense, 10: 3.

Wheeler, B. E. J. 1969. An Introduction to Plant Diseases. John Wiley and Sons Ltd., London. p.301.

Wilcoxson, R. D., Skovmand, B., and Atif, A. H. 1975. Evaluation of wheat cultivars for ability to retard development of stem rust. Ann. Appl. Biol., 80: 275-281.

\section{How to cite this article:}

Meghana, S. P., S. I. Harlapur, P.V. Patil and Kachapur, R. M. 2020. Assessment of fungicide spray schedules against turcicum leaf blight of maize caused by Exserohilum turcicum (Pass.) Leonard and Suggs. Int.J.Curr.Microbiol.App.Sci. 9(05): 646-655. doi: https://doi.org/10.20546/ijcmas.2020.905.071 